Article

\title{
Synthesis and Biological Screening of 4-Benzyl-2H-phthalazine Derivatives
}

Ashraf H.F. Abd El-Wahab ${ }^{1,2, *}$, Hany M. Mohamed ${ }^{1,3}$, Ahmed M. El-Agrody ${ }^{4}$, Mohammed A. El-Nassag ${ }^{1}$ and Ahmed H. Bedair ${ }^{1}$

1 Chemistry Department, Faculty of Science, Al-Azhar University, 11884, Nasr City, Cairo, Egypt

2 Chemistry Department, Faculty of Science, Jazan University, 2097, Jazan, Saudi Arabia

3 Chemistry Department, Faculty of Medicine, Jazan University, 82621, Jazan, Saudi Arabia

4 Chemistry Department, Faculty of Science, King Khalid University, 9004, Abha, Saudi Arabia

* Author to whom correspondence should be addressed; E-Mail: ash_abdelwahab@hotmail.com.

Received: 20 June 2011; in revised form: 13 July 2011 / Accepted: 13 July 2011 /

Published: 17 August 2011

\begin{abstract}
Preparation of 4-benzyl-2-substituted phthalazin-1-one derivatives 2-8 is reported. Condensation of 4-benzyl-1-chlorophthalazine (9) with a series of different nucleophiles gave 4-benzylphthalazin-1-ylamino derivatives (10-13 and 16) and 4-amino-2-[N"-(4-benzylphthalazin-1-yl)-hydrazino]-6-arylpyrimidine-5-carbonitriles (14a,b). Interaction of 9 with ambident anions was also studied. 5-Benzyl-6,6a,12-triazobenzo[a]anthracen-7-one (15) is obtained from 9 and anthranilic acid derivatives. Treatment of 16 with $(\mathrm{EtO})_{3} \mathrm{CH} / \mathrm{Ac}_{2} \mathrm{O}$ under reflux afforded the corresponding ethoxymethylene derivative 17, while aqueous ammonium hydroxide treatment afforded carboxamide derivative $\mathbf{1 8}$. The structures of the newly synthesized derivatives were confirmed by their elemental analysis, IR, ${ }^{1} \mathrm{H}$ NMR, ${ }^{13} \mathrm{C}$ NMR and mass spectral studies. Antimicrobial activities of some selected compounds were also studied and some of these were found to exhibit promising effects against Gram-positive and Gram-negative bacteria and fungi.
\end{abstract}

Keywords: phthalazine derivatives; anthracene derivatives; synthesis; antimicrobial activity 


\section{Introduction}

The phthalazine derivative azelastine (A, Figure 1) is an antihistamine used in the treatment of allergic rhinitis [1]. Newer agents are more selective inhibitors of the cGMP-inhibited phosphor diesterase (PDE) and casn be exemplified by phthalazine derivatives like MY5445 (B, Figure 1) [2-5]. Zopolrestat (C, Figure 1) is a phthalazinone derivative that has been in clinical trials; it inhibits aldose reductase and has potential use in the prevention of retinopathy, neuropathy, and cataract formation in diabetes [6]. The chemiluminescence reactions of luminol (D, Figure 1) and related phthalazines have found analytical applications, particularly in biological systems where the inherent signal strength and low signal noise ratio contribute to sensitivity. The hydrogen peroxide/luminol system has been used for the on-line determination by chemiluminescence of nitric oxide in isolated organ perfusates [7-10].

Figure 1. Phthalazine derivatives (azelastin, MY 5445, zopolrestat and luminol).<smiles>CN1CCCC(Cn2nc(Cc3ccc(Cl)cc3)c3ccccc3c2=O)CC1</smiles>

A<smiles>O=C(O)Cc1nn(Cc2nc3cc(C(F)(F)F)ccc3s2)c(=O)c2ccccc12</smiles>

C<smiles>Clc1cccc(Nc2nnc(-c3ccccc3)c3ccccc23)c1</smiles>

B<smiles>Nc1cccc2c(=O)[nH]nc(O)c12</smiles>

D

Phthalazine derivatives have been widely applied as therapeutic agents due to their anticonvulsant, cardiotonic, vasorelaxant and anti-inflammatory properties [11-17] in addition to having antimicrobial activity [18].

\section{Results and Discussion}

4-Benzyl-2H-phthalazin-1-one (1) [19] was used as a starting material for this investigation. Reaction of compound 1 with formalin in ethanol afforded 4-benzyl-2-hydroxymethyl-2H-phthalazin-1-one (2). Treatment of $\mathbf{2}$ with thionyl chloride afforded the corresponding 4-benzyl-2-chloromethyl- $\mathrm{H}$ phthalazin-1-one (3). Interaction of $\mathbf{3}$ with alcoholic potassium thiocyanate yield the corresponding 4-benzyl-2-thiocyanatomethyl-2H-phthalazin-1-one (4), while with ethanolic thiourea it afforded 4-benzyl-2-mercaptomethyl-2H-phthalazin-1-one (5). Treatment of 1 with ethyl chloroacetate in the presence of anhydrous $\mathrm{K}_{2} \mathrm{CO}_{3}$ afforded the corresponding ethyl (2-(4-benzyl-1-oxo-1H-phthalazin-2-yl)acetate (6). 
Reaction of 6 with hydrazine hydrate gave the corresponding (4-benzyl-1-oxo-1H-phthalazin-2-yl)acetic acid hydrazide (7) which was reacted with $p$-tolualdehyde to give the corresponding (4-benzyl-1-oxo-1H-phthalazin-2-yl) acetic acid (4-methylbenzylidene) hydrazide (8) (Scheme 1).

Scheme 1. Synthesis of phthalazine-1-one derivatives.<smiles>O=c1[nH]nc(Cc2ccccc2)c2ccccc12</smiles>

1<smiles>[Y]C(=O)OCCC(=O)OCC</smiles><smiles>CCOC(=O)Cn1nc(Cc2ccccc2)c2ccccc2c1=O</smiles>

6<smiles>NNC(=O)Cn1nc(Cc2ccccc2)c2ccccc2c1=O</smiles>
7<smiles>CCOC(C)c1ccc2c(Cc3ccccc3)nn(CCl)c(=O)c2c1</smiles>

2

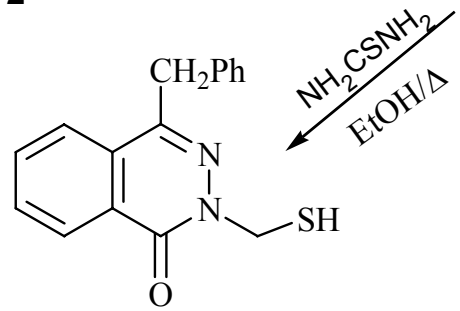<smiles>Cc1ccc(C=NNC(=O)Cn2nc(Cc3ccccc3)c3ccccc3c2=O)cc1</smiles>

4-Benzyl-1-chlorophthalazine (9) [19] was used as starting martial from preparation of new 1,4-disubstuted phthalazine derivatives. Thus, interaction of 9 with equimolar amount of aminophenols, aminoacetophenones and $p$-aminobenzoic acid gave the corresponding 4-benzylphthalazin-1-ylamino derivatives (10a-f). Compound 9 also reacted with $N$-(4-aminophenyl)acetanilide under reflux to give the corresponding 4-(4-benzylphthalazin-1-ylamino)acetanilide (10g), in the case of $p$-phenylene-diamine and/or benzidine but in the case of $p$-aminodiphenyl amine the only isolable product is 4-(4-benzylphthalazin-1-ylamino)diphenylamine (10h). Treatment of 9 with sulfanilic acid and/or J-acid (2-amino-5-naphthol-7-sulfonic acid) gave the corresponding 4-(4-benzylphthala-zin-1ylamino)benzenesulfonic acid (10i) and/or 7-(4-benzylphthalazin-1-ylamino)-4-hydroxynaphthalene2-sulfonic acid (10j), while $\mathbf{9}$ with ammonium thiocyanate gave the corresponding 4-benzylphthalazin-1-ylthiocyanate (11). Interaction of 9 with dibasic aromatic amines in a 1:2 ratio under fusion conditions afforded the corresponding $N, N^{\prime}$-bis(4-benzyl-phthalazin-1-yl)-1,4-phenylenediamine 
and/or $N, N^{\prime}$-bis(4-benzylphthalazin-1-yl)biphenyl-4,4'-diamines (12a,b). Also, 9 was reacted with ethylenediamine in boiling ethanol to give $N, N^{\prime}$-bis(4-benzylphthalazin-1-yl)ethane-1,2-diamine (13), However, interaction of 9 with 2-hydrazino-4-amino-5-cyano-6-arylpyrimidine gave the corresponding pyrimidine-5-carbonitrile derivatives 14a,b respectively (Scheme 2). Interaction of 9 with methyl anthranilate and/or anthranilic acid gave anthracen-7-one derivative $\mathbf{1 5}$ as the only isolable product. The formation of $\mathbf{1 5}$ was explained by the formation of intermediate [E] which undergoes intramolecular ring closure to the final product 15 (Scheme 3).

Scheme 2. Reactions of 4-benzyl-1-chlorophthalazine with amines derivatives.

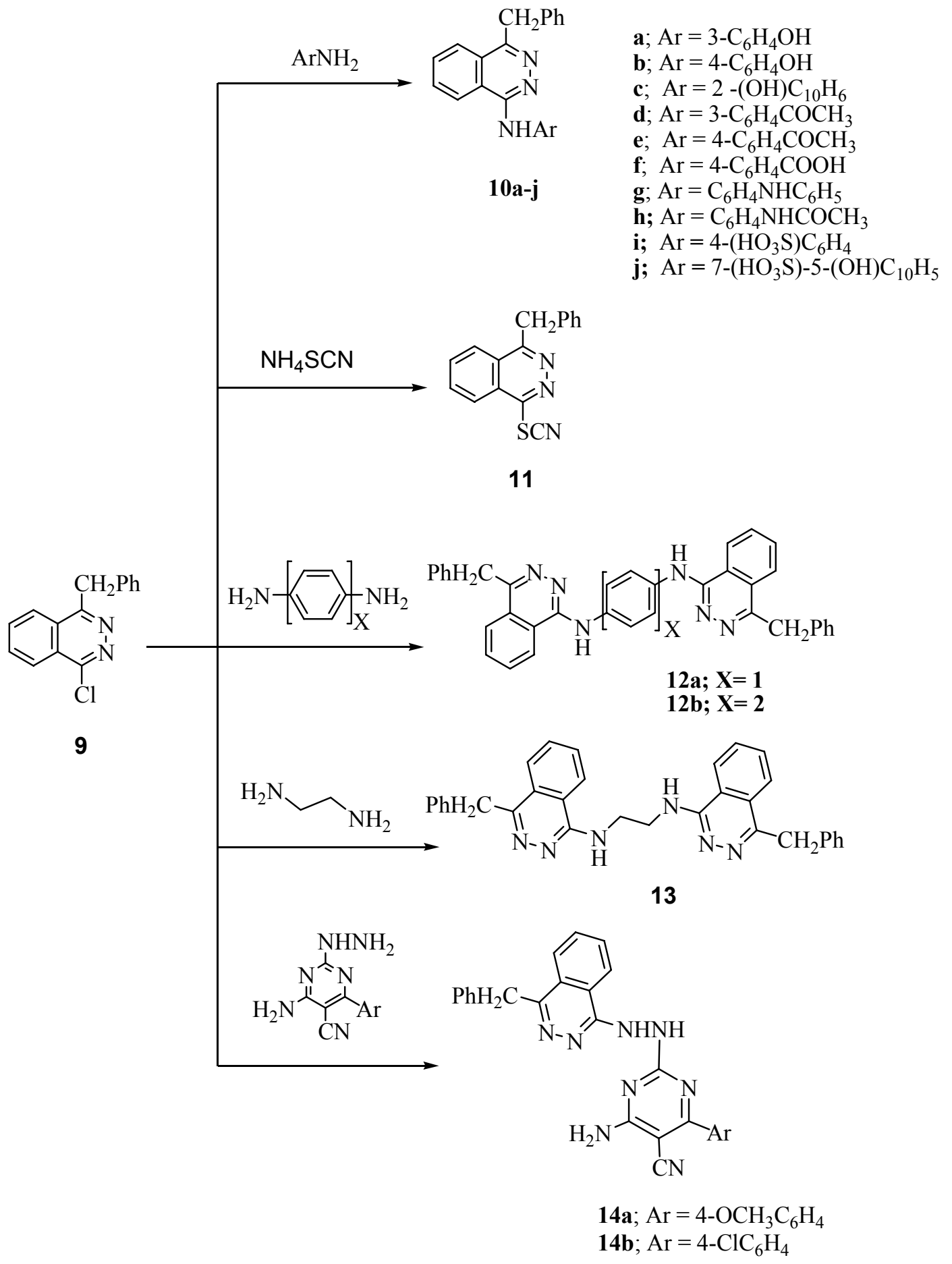


Treatment of 9 with $p$-aminohippuric acid in boiling methanol/TEA, gave the corresponding methyl ester derivative 16 was obtained as the only isolable product. The formation of $\mathbf{1 6}$ can be explained by the nucleophilic transformation into benzylphthalazin-1-ylamino derivative $[\mathbf{F}]$ as intermediate which undergoes intramolecular cyclization into oxazolone intermediate $[\mathbf{G}]$ then reacted with a molecule of methanol to give the methyl ester derivative $\mathbf{1 6}$ as the final product (Scheme 3).

Scheme 3. Synthesis of triazobenzobenzo[a]anthrcen-7-one, methyl ester and carboxamide derivatives.

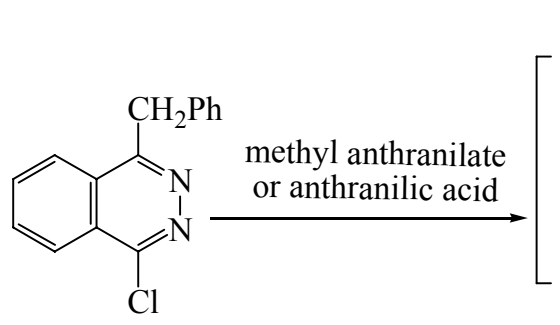

9

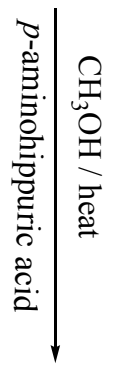

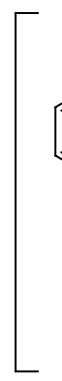

$[\mathbf{F}]$<smiles>O=C(O)CCc1nnc(Cc2ccccc2)c2ccccc12</smiles>

$[\mathbf{E}]$<smiles>O=c1c2ccccc2nc2c3ccccc3c(Cc3ccccc3)nn12</smiles>

15

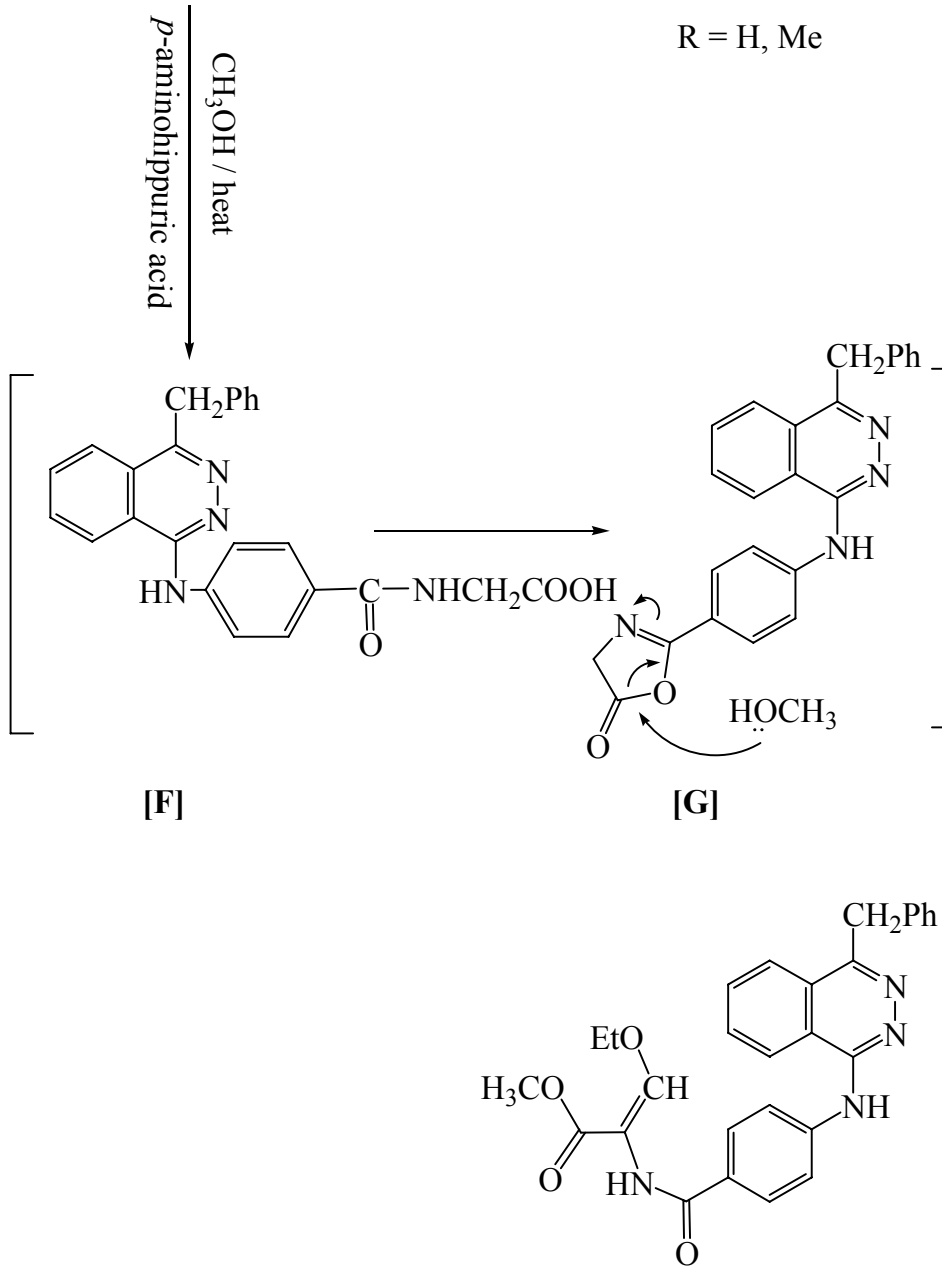

$\mathrm{R}=\mathrm{H}, \mathrm{Me}$

17<smiles>COC(=O)CNC(=O)c1ccc(Nc2nnc(Cc3ccccc3)c3ccccc23)cc1</smiles><smiles>NC(=O)CNC(=O)c1ccc(Nc2nnc(Cc3ccccc3)c3ccccc23)cc1</smiles>

18

Treatment of 16 with $(\mathrm{EtO})_{3} \mathrm{CH} / \mathrm{Ac}_{2} \mathrm{O}$ under reflux afforded the corresponding ethoxymethylene derivative 17. Treatment of $\mathbf{1 6}$ with aqueous ammonium hydroxide under reflux in dioxane in the presence of triethylamine afforded the corresponding carboxamide derivative $\mathbf{1 8}$ respectively (Scheme 3). 


\section{Experimental}

\subsection{General}

Melting points were determined on a Stuart melting point apparatus and are uncorrected. IR spectra were recorded in $\mathrm{KBr}$ using a FT-IR 5300 spectrometer and Perkin Elmer spectrum RXIFT-IR system $\left(v, \mathrm{~cm}^{-1}\right)$. The ${ }^{1} \mathrm{H}$ NMR at $(300 \mathrm{MHz})$ and ${ }^{13} \mathrm{C}$ NMR spectra $(75 \mathrm{MHz})$ were recorded in $\mathrm{CDCl}_{3}$ or DMSO- $d_{6}$ on a Varian Mercury VX-300 NMR spectrometer. Chemical shifts $(\delta)$ are related to that of the solvent. Mass spectra were measured on a Shimadzu GMMS-QP-1000 EX mass spectrometer at $70 \mathrm{eV}$. The elemental analyses were performed at the Microanalytical Center, Cairo University. Cairo (Egypt).

4-Benzyl-2-hydroxymethyl-2H-phthalazin-1-one (2). A mixture of compound 1 (0.23 g, $1.0 \mathrm{mmol})$ and formaldehyde solution $38 \%(0.8 \mathrm{~mL})$ in ethanol $(30 \mathrm{~mL})$ was refluxed for 3 hours. The solvent was evaporated under vacuum, and then water $(25 \mathrm{~mL})$ was added. The solid obtained was filtered off and recrystallized from ethanol. Colourless crystals, $90 \%, 0.19$ g. mp $115-116{ }^{\circ} \mathrm{C}$; IR $\left(v_{\max }, \mathrm{cm}^{-1}\right): 3334$ $(\mathrm{OH}), 1640(\mathrm{CO}) .{ }^{1} \mathrm{H}$ NMR $\left(\mathrm{CDCl}_{3}\right): \delta_{\mathrm{H}} 4.13(\mathrm{t}, J=8.4 \mathrm{~Hz}, 1 \mathrm{H}, \mathrm{OH}), 4.31\left(\mathrm{~s}, 2 \mathrm{H}, \mathrm{CH}_{2} \mathrm{Ph}\right), 5.69(\mathrm{~d}$, $\left.J=8.4 \mathrm{~Hz}, 2 \mathrm{H}, \mathrm{CH}_{2} \mathrm{OH}\right), 7.30(\mathrm{~m}, 5 \mathrm{H}, \mathrm{Ph}-\mathrm{H}), 7.71(\mathrm{~m}, 3 \mathrm{H}$, phthalazinyl-3H), $8.45(\mathrm{~m}, 1 \mathrm{H}$, phthalazinyl-1H); the singlet at 4.134 was cancelled by $\mathrm{D}_{2} \mathrm{O} .{ }^{13} \mathrm{C}$ NMR $\left(\mathrm{DMSO}-\mathrm{d}_{6}\right) \delta 37.3\left(\mathrm{CH}_{2}\right), 60.1$ $\left(\mathrm{CH}_{2} \mathrm{OH}\right), 125.7\left(\mathrm{C}_{4}-\mathrm{Ar}\right), 128\left(\mathrm{C}_{5}\right), 128.3\left(\mathrm{C}_{8}\right), 128.8\left(\mathrm{C}_{3}-\mathrm{Ar}, \mathrm{C}_{5}-\mathrm{Ar}\right), 129.1\left(\mathrm{C}_{2}-\mathrm{Ar}, \mathrm{C}_{6}-\mathrm{Ar}\right), 130.2$ $\left(\mathrm{C}_{5 \mathrm{a}}\right), 130.3\left(\mathrm{C}_{8 \mathrm{a}}\right), 131.2\left(\mathrm{C}_{7}\right), 132.3\left(\mathrm{C}_{6}\right), 137.2\left(\mathrm{C}_{1}-\mathrm{Ar}\right), 155.1\left(\mathrm{C}_{4}\right)$ and $160.1(\mathrm{CO}) . \mathrm{MS}$, $m / z(\%)=266\left(\mathrm{M}^{+}, 10.25\right), 235(100)$ together with other peaks at 207 (5.74), $178(20.38), 149$ (15.82), 130 (9.19), 91 (12.03), 57 (17.77); Anal. Calcd for $\mathrm{C}_{16} \mathrm{H}_{14} \mathrm{~N}_{2} \mathrm{O}_{2}$ (266.10): C, 72.16; H, 5.30; N, $10.52 \%$. Found: C, 71.89; H, 5.02; N, 9.96\%.

4-Benzyl-2-chloromethyl-2H-phthalazin-1-one (3). A mixture of compound 2 (0.26 g, $1.0 \mathrm{mmol})$ and thionyl chloride $(5 \mathrm{~mL})$ was refluxed for 1 hour. The solid obtained was filtered off and recrystallized from benzene. Colourless crystals, 87\%, 0.12 g. mp 125-127 ${ }^{\circ} \mathrm{C}$; IR ( $\left.v_{\max }, \mathrm{cm}^{-1}\right): 3046$ (C-H aromatic), $1662(\mathrm{CO})$. MS, $m / z(\%)=284\left(\mathrm{M}^{+}, 43.4\right), \mathrm{M}+2(12.7), 249(100)$ together with other peaks at 220 (34.9), 178 (17.9), 130 (29.2), 91 (80.7), 51 (28.9); Anal. Calcd for $\mathrm{C}_{16} \mathrm{H}_{13} \mathrm{ClN}_{2} \mathrm{O}$ (284.07): C, 67.49; H, 4.60; N, 9.84\%. Found: C, 66.90; H, 4.08; N, 9.11\%.

4-Benzyl-2-thiocyanatomethyl-2H-phthalazin-1-one (4). A mixture of compound 3 (0.28 g, $1.0 \mathrm{mmol})$ and potassium thiocyanate $(1.0 \mathrm{mmol})$ in ethanol $(30 \mathrm{~mL})$ was refluxed for 3 hours. The solvent was evaporated under vacuum. The solid obtained was filtered off and recrystallized from ethanol. White crystals, 85\%, 0.17 g. mp 155-156 ${ }^{\circ} \mathrm{C}$; IR $\left(v_{\max }, \mathrm{cm}^{-1}\right): 2158(\mathrm{SCN}), 1664(\mathrm{CO}) . \mathrm{MS}, \mathrm{m} / z(\%)=307$ $\left(\mathrm{M}^{+}, 0.7\right), 249$ (56.3), 130 (12.8) and 91 (100); Anal. Calcd for $\mathrm{C}_{17} \mathrm{H}_{13} \mathrm{~N}_{3} \mathrm{OS}$ (307.36): C, 66.43; $\mathrm{H}$, 4.26 ; N, 13.67\%. Found: C, 66.22; H, 3.98; N, 13.50\%.

4-Benzyl-2-mercaptomethyl-2H-phthalazin-1-one (5). A mixture of compound 3 (0.28 g, $1.0 \mathrm{mmol})$ and thiourea $(1.0 \mathrm{mmol})$ in ethanol $(30 \mathrm{~mL})$ was refluxed for $3 \mathrm{~h}$ hours. The solvent was evaporated under vacuum. The solid obtained was filtered off and recrystallized from ethanol. White crystals, $80 \%, 0.10$ g. $\mathrm{mp} 145-146^{\circ} \mathrm{C}$; IR $\left(v_{\max }, \mathrm{cm}^{-1}\right): 2750(\mathrm{SH}), 1650(\mathrm{CO}) . \mathrm{MS}, m / z(\%)=282\left(\mathrm{M}^{+}, 17.2\right)$, 
249 (78.2), 235 (18.1), 132 (27.3), 130 (23.5), 91 (100) 51 (32.8); Anal. Calcd for $\mathrm{C}_{16} \mathrm{H}_{14} \mathrm{~N}_{2} \mathrm{OS}$ (282.36): C, 68.06; H, 5.00; N, 9.92\%. Found: C, 67.45; H, 4.67; N, 9.22\%.

Ethyl (4-benzyl-1-oxo-1H-phthalazin-2-yl)acetate (6). A mixture of compound 1 (0.23 g, $10 \mathrm{mmol})$ and ethyl chloroacetate $(2 \mathrm{~mL})$ and anhydrous $\mathrm{K}_{2} \mathrm{CO}_{3}(0.13 \mathrm{~g}, 1.0 \mathrm{mmol})$ was refluxed for 4 hours. The solvent was evaporated under vacuum, then water $(50 \mathrm{~mL})$ was added. The solid obtained was filtered off and recrystallized from pet. ether and chloroform, respectively. Violet crystals, 65\%, $0.13 \mathrm{~g}$. $\mathrm{mp}$ 76-78 ${ }^{\circ} \mathrm{C}$; IR ( $\left.v_{\max }, \mathrm{cm}^{-1}\right)$ : 2978 (CH aliphatic), 1744, $1648(\mathrm{CO}) . \mathrm{MS}, m / z(\%)=322\left(\mathrm{M}^{+}, 37.9\right)$ and base peak at 248 together with other peak at 249 (97.9), 221 (8.2), 220 (21.5), 219 (24.3), 193 (4.6), 102 (14.6), 91 (98.4), 76 (11.4); Anal. Calcd for $\mathrm{C}_{19} \mathrm{H}_{18} \mathrm{~N}_{2} \mathrm{O}_{3}$ (322.12): C, 70.79; H, 5.63; N, 8.69\%. Found: C, 70.36; H, 5.44; N, 8.52\%.

(4-Benzyl-1-oxo-1H-phthalazin-2-yl)acetic acid hydrazide (7). A mixture of compound 6 (0.32 g, $10 \mathrm{mmol})$ and hydrazine hydrate $(0.8 \mathrm{~mL})$ in ethanol $(30 \mathrm{~mL})$ was refluxed for 3 hours. The solvent was evaporated under vacuum, then water $(25 \mathrm{~mL})$ was added. The solid obtained was filtered off and recrystallized from ethanol. White crystals, $86 \%, 0.18$ g. mp $130-132{ }^{\circ} \mathrm{C}$; IR $\left(v_{\max }, \mathrm{cm}^{-1}\right): 3326(\mathrm{NH})$, $1684\left(\mathrm{CO}\right.$, carboxylic acid hydrazide), $1642\left(\mathrm{CO}\right.$, phthalazinyl). ${ }^{1} \mathrm{H} \mathrm{NMR}\left(\mathrm{CDCl}_{3}\right): \delta_{\mathrm{H}} 14.29(\mathrm{~s}, 2 \mathrm{H}$, $\left.\mathrm{CH}_{2} \mathrm{Ph}\right), 4.75$ (s, 2H, NCH${ }_{2} \mathrm{CO}$ ), 7.29 (m, 5H, Ar-H), 7.81 (m, 5H, phthalazinyl-3H and $\left.\mathrm{NH}_{2}\right), 8.25$ (m, $1 \mathrm{H}$, phthalazinyl-H) and $9.30\left(\mathrm{~s}, 1 \mathrm{H}, \mathrm{CONH}\right.$; cancelled by $\left.\mathrm{D}_{2} \mathrm{O}\right) . \mathrm{MS}, \mathrm{m} / z(\%)=308\left(\mathrm{M}^{+}, 0\right), 278$ (7.2), 277 (23.4), 249 (44.9), 130 (15.3), 91 (100); Anal. Calcd for $\mathrm{C}_{17} \mathrm{H}_{16} \mathrm{~N}_{4} \mathrm{O}$ (308.33): C, 66.22; $\mathrm{H}$, $5.23 ; \mathrm{N}, 18.17 \%$. Found: C, 66.09; H, 5.12; N, 17.89\%.

\subsection{General Procedure for the Synthesis of Benzylphthalazin-1-ylamino Derivatives 10a-h}

A mixture of compound $9(0.25 \mathrm{~g}, 10 \mathrm{mmol})$ and aromatic amine $(10 \mathrm{mmol})$ in ethanol $(30 \mathrm{~mL})$ was refluxed for 3 hours. The solvent was evaporated under vacuum. The solid obtained was filtered off to give crude products

(4-Benzylphthalazin-1-ylamino)phenols (10a,b). Compound 10a: Yellow crystals, 90\%, 0.17 g. mp 240-242 ${ }^{\circ} \mathrm{C}$; IR $\left(v_{\max }, \mathrm{cm}^{-1}\right): 3258\left(\mathrm{NH}\right.$ and $\mathrm{OH}$ phenolic) as broad peak. MS, $m / z(\%)=307327\left(\mathrm{M}^{+}\right.$, 42.2) and the base peak at 326 together with other peaks at 325 (84.3), 91 (62.6); Anal. Calcd for $\mathrm{C}_{21} \mathrm{H}_{17} \mathrm{~N}_{3} \mathrm{O}$ (327.10): C, 77.04; H, 5.23; N, 12.84\%. Found: C, 76.83; H, 5.02; N, 12.56\%. Compound 10b: Yellow crystals, $80 \%, 0.12 \mathrm{~g}$. $\mathrm{mp} 236-237^{\circ} \mathrm{C}$; IR $\left(v_{\max }, \mathrm{cm}^{-1}\right): 3268$ (NH and $\mathrm{OH}$ phenolic) as broad peak. MS, $m / z(\%)=327\left(\mathrm{M}^{+}, 40.0\right)$ and the base peak at 326 together with other peaks at 325 (86.7), 91 (50.8); Anal. Calcd for $\mathrm{C}_{21} \mathrm{H}_{17} \mathrm{~N}_{3} \mathrm{O}$ (327.10): C, 77.04; H, 5.23; N, 12.84\%. Found: C, 76.75; $\mathrm{H}, 5.01 ; \mathrm{N}, 12.59 \%$.

7-(4-Benzylphthalazin-1-ylamino)naphthalen-2-ol (10c). Green crystals, 70\%, 0.09 g. mp 242-243 ${ }^{\circ} \mathrm{C}$; IR $\left(v_{\max }, \mathrm{cm}^{-1}\right): 3272(\mathrm{OH}$ and $\mathrm{NH})$ as broad peak. ${ }^{1} \mathrm{H}$ NMR (DMSO- $\left.d_{6}\right): \delta_{\mathrm{H}} 24.65\left(\mathrm{~s}, 2 \mathrm{H}, \mathrm{CH}_{2} \mathrm{Ph}\right)$, 7.08-7.33, 7.55-7.58, 8.09-8.11 (ms, 9H, Ar-H), 7.38 (d, $J=7.8 \mathrm{~Hz}, 1 \mathrm{H}, \mathrm{Ar}-\mathrm{H}), 7.78$ (d, J=9 Hz, 1H, Ar-H), $7.86(\mathrm{~d}, J=9 \mathrm{~Hz}, 1 \mathrm{H}, \mathrm{Ar}-\mathrm{H}), 8.14$ (d, $J=1.8 \mathrm{~Hz}, 1 \mathrm{H}, \mathrm{Ar}-\mathrm{H}), 8.16$ (s, 1H, NH, exchangeable with $\left.\mathrm{D}_{2} \mathrm{O}\right), 8.33(\mathrm{~d}, J=1.8 \mathrm{~Hz}, 1 \mathrm{H}, \mathrm{Ar}-\mathrm{H}), 9.04(\mathrm{~d}, J=7.8 \mathrm{~Hz}, 1 \mathrm{H}, \mathrm{Ar}-\mathrm{H})$ and $9.96(\mathrm{~s}, 1 \mathrm{H}$, 
phenolic-OH, exchangeable with $\mathrm{D}_{2} \mathrm{O}$ ).; Anal. Calcd for $\mathrm{C}_{25} \mathrm{H}_{19} \mathrm{~N}_{3} \mathrm{O}$ (377.13): C, 79.55; H, 5.07; N, 11.13\%. Found: C, 79.32; H, 4.87; N, 10.92\%.

1-[(4-Benzylphth-alazin-1-ylamino)phenyl]ethanones (10d,e). Compound 10d: White crystals, 75\%, 0.11 g. mp $225-226{ }^{\circ} \mathrm{C}$; IR $\left(v_{\max }, \mathrm{cm}^{-1}\right): 3390(\mathrm{NH}), 1662(\mathrm{CO}) . \mathrm{MS}, \mathrm{m} / z(\%)=353(73.56)$ and the base peak at 352 together with other peaks at 311 (3.7), 310 (11.97), 262 (10.56), 220 (5.37), 219 (4.82) and 91 (54.14); Anal. Calcd for $\mathrm{C}_{23} \mathrm{H}_{19} \mathrm{~N}_{3} \mathrm{O}$ (353.05): C, 78.16; H, 5.42; N, 11.89\%. Found: C, 77.88; H, 5.23; N, 11.64\%. Compound 10e: Yellow crystals, $80 \%, 0.13$ g. mp 260-261 ${ }^{\circ} \mathrm{C}$; IR $\left(v_{\max }\right.$, $\mathrm{cm}^{-1}$ ): $3312(\mathrm{NH}), 1670(\mathrm{CO}) .{ }^{1} \mathrm{H}$ NMR (DMSO-d $\left.)_{6}\right): \delta_{\mathrm{H}} 2.57\left(\mathrm{~s}, 3 \mathrm{H}, \mathrm{CH}_{3} \mathrm{CO}\right), 4.75\left(\mathrm{~s}, 2 \mathrm{H}, \mathrm{CH}_{2} \mathrm{Ph}\right)$, 7.21-7.27 (m, 2H, Ar-H), 7.31 and 7.40 (AB-q, J=7.2 Hz, 4H, Ar-H), 8.01 (s, 1H, NH), 8.10-8.18 (m, $5 \mathrm{H}, \mathrm{Ar}-\mathrm{H})$ and 8.39, $8.94(2 \mathrm{~d}, J=7.8 \mathrm{~Hz}, 2 \mathrm{H}, \mathrm{Ar}-\mathrm{H})$; Anal. Calcd for $\mathrm{C}_{23} \mathrm{H}_{19} \mathrm{~N}_{3} \mathrm{O}$ (353.23): C, 78.18; H, 5.42; N, 11.89\%. Found: C, 77.97; H, 5.18; N, 11.69\%.

4-(4-Benzylphthalazin-1-ylamino)benzoic acid (10f). Yellow crystals, 85\%, 0.16 g. mp 280-281 ${ }^{\circ} \mathrm{C}$; IR $\left(v_{\max }, \mathrm{cm}^{-1}\right): 3224(\mathrm{NH}$ and $\mathrm{OH}), 1676(\mathrm{CO}) . \mathrm{MS}, m / z(\%)=355(38.4)$ and base peak at 354 together with other peaks at 310 (3), 234 (5.3), 204 (9.9), 219 (6.9), 204 (9.9), 178 (10.1), 102 (15.9), 91 (68.3), 90 (60.2), 77 (30.6) and 76 (29.7); Anal. Calcd for $\mathrm{C}_{22} \mathrm{H}_{17} \mathrm{~N}_{3} \mathrm{O}_{2}$ (355.16): C, 74.35; H, 4.82; N, $11.82 \%$. Found: C, 74.14; H, 4.57; N, 11.60\%.

4-(4-Benzylphthalazin-1-ylamino)diphenyl amine (10g). Green crystals, 70\%, 0.13 g. mp 215-217 ${ }^{\circ} \mathrm{C}$; IR $\left(v_{\max }, \mathrm{cm}^{-1}\right): 3276(\mathrm{NH}) . \mathrm{MS}, m / z(\%)=402\left(\mathrm{M}^{+}, 46.5\right), 117$ (88.7), 92 (60.32), 76 (28.37), 91 (18.88), 57 (87.49), 56 (29.88) \& 55 (100); Anal. Calcd for $\mathrm{C}_{27} \mathrm{H}_{22} \mathrm{~N}_{4}(402.02): \mathrm{C}, 80.57$; H, 5.51; N, 13.92\%. Found: C, 80.44; H, 5.49; N, 13.75\%.

4-(4-benzylphthalazin-1-ylamino)acetanilide (10h). Yellow crystals, 80\%, $0.18 \mathrm{~g} . \mathrm{mp}>340{ }^{\circ} \mathrm{C}$; IR $\left(v_{\max }, \mathrm{cm}^{-1}\right): 3428(\mathrm{NH}), 1620(\mathrm{CO}) . \mathrm{MS}, \mathrm{m} / z(\%)=368\left(\mathrm{M}^{+}, 12.94\right), 235$ (10.25), 149 (88.35), 117 (78.7), 92 (63.32), 76 (28.37), 91 (18.88), 57 (87.49), 56 (29.88) and 55 (100); Anal. Calcd for $\mathrm{C}_{23} \mathrm{H}_{20} \mathrm{~N}_{4} \mathrm{O}$ (368.17): C, 74.98; H, 5.47; N, 15.21\%. Found: C, 74.80; H, 5.32; N, 15.12\%.

\subsection{General Method for the Synthesis of Sulfonic Acid Derivatives $\mathbf{1 0 i}, \mathbf{j}$}

A mixture of compound $9(0.25 \mathrm{~g}, 1.0 \mathrm{mmol})$ in ethanol $(30 \mathrm{~mL})$ sulfanilic acid or (2-amino-5-naphthol-7-sulfonic acid) $(1.0 \mathrm{mmol})$ and 2 drops of triethylamine (TEA) was refluxed for 3 hours. The solvent was evaporated under vacuum. The solid obtained was filtered off and recrystallized from dioxane.

4-(4-Benzylphthalazin-1-ylamno)benzenesulfonic acid (10i). Yellow crystals, 80\%, $0.17 \mathrm{~g}$. $\mathrm{mp}>340^{\circ} \mathrm{C}$; IR $\left(v_{\max }, \mathrm{cm}^{-1}\right): 3402(\mathrm{OH}), 3256(\mathrm{NH}) . \mathrm{MS}, m / z(\%)=391\left(\mathrm{M}^{+}, 4.7\right), 327(18.12), 311$ (72.15), 310 (95.64), 235 (40.60), 219 (89.60), 193 (100), 92 (57.72) and 91 (16.78). Anal. Calcd for $\mathrm{C}_{21} \mathrm{H}_{17} \mathrm{~N}_{3} \mathrm{O}_{3} \mathrm{~S}$ (391.09): C, 64.43; H, 4.38; N, 10.73\%. Found: C, 64.36; H, 4.28; N, 10.65\%.

7-(4-benzylphthalazin-1-ylamino)-4-hydroxynaphthalene-2-sulfonic acid (10j). Yellow crystals, 65\%, 0.13 g. mp $>340{ }^{\circ} \mathrm{C}$; IR $\left(v_{\max }, \mathrm{cm}^{-1}\right): 3308(\mathrm{OH}), 3066(\mathrm{NH}) . \mathrm{MS}, m / z(\%)=457\left(\mathrm{M}^{+}, 5.3\right), 455$ 
(naphthsaltone cationic radical, 59.1), 222 (5.1), 235 (100), 203 (6.4), 193 (3.6) and 178 (15.8); Anal. Calcd for $\mathrm{C}_{25} \mathrm{H}_{19} \mathrm{~N}_{3} \mathrm{O}_{4} \mathrm{~S}$ (457): C, 65.63; H, 4.19; N, 9.18\%. Found: C, 65.54; H, 3.97; N, 9.10\%.

Synthesis of N,N'-bis(4-benzylphthalazin-1-yl)-1,4-phenylenediamine (11) and N,N'-bis(4benzylphthalazin-1-yl)biphenyl-4,4'-diamine (12). A mixture of compound 9 (0.50 g, $20 \mathrm{mmol})$ and dibasic aromatic amines $(10 \mathrm{mmol})$ in ethanol $(30 \mathrm{~mL})$ was refluxed for 3 hours. The solvent was evaporated under vacuum. The solid obtained was filtered off and recrystallized from dioxane. Compound 11 Yellow crystals, 70\%, 0.13 g. mp 250-251 ${ }^{\circ}$; IR $\left(v_{\max }, \mathrm{cm}^{-1}\right): 3344(\mathrm{NH}) . \mathrm{MS}$, $m / z(\%)=544\left(\mathrm{M}^{+}, 0.74\right)$ and base peak at 325 together with other peaks at $234(5.7), 219(3.29), 106$ (1.74), 91 (13.66), 76 (1.25); Anal. Calcd for $\mathrm{C}_{36} \mathrm{H}_{28} \mathrm{~N}_{6}$ (544.27): C, 79.39; H, 5.18; N, 15.43\%. Found: C, 79.08; H, 5.06; N, 15.22\%. Compound 12: Yellow crystals, 70\%, 0.12 g. $\mathrm{mp}>340{ }^{\circ} \mathrm{C}$; IR ( $v_{\max }$, $\left.\mathrm{cm}^{-1}\right): 3277(\mathrm{NH})$. MS, $m / z(\%)=620\left(\mathrm{M}^{+}, \mathrm{O}\right), \mathrm{M} / 2(310,9.3)$; the base peak at 401 and other peaks at 102 (1.8), 91 (17.1); Anal. Calcd for $\mathrm{C}_{42} \mathrm{H}_{32} \mathrm{~N}_{6}$ (620.13): C, 81.27; H, 5.20; N, 13.54\%. Found: C, $80.96 ; \mathrm{H}, 5.04 ; \mathrm{N}, 13.30 \%$.

$N, N^{\prime}$-bis(4-benylphthalazin-1-yl)ethane-1,2-diamine (13). A mixture of compound 9 (0.50 g, $\left.20 \mathrm{mmol}\right)$ and ethylenediamine $(10 \mathrm{mmol})$ in absolute ethanol $(30 \mathrm{~mL})$ anhydrous $\mathrm{K}_{2} \mathrm{CO}_{3}$ was refluxed for 4 hours. The obtained was cooled to room temperature, then diluted with water. The solid obtained was filtered off and recrystallized from ethanol. White crystals, $60 \%, 0.21 \mathrm{~g} . \mathrm{mp} 234-235{ }^{\circ} \mathrm{C}$; IR $\left(v_{\max }\right.$, $\mathrm{cm}^{-1}$ ): 3316, $3260(\mathrm{NH})$ 2930, 2870 (C-H aliphatic). ${ }^{1} \mathrm{H}$ NMR (DMSO- $\left.d_{6}\right): \delta_{\mathrm{H}} 3.93\left(\mathrm{~s}, 4 \mathrm{H}, \mathrm{CH}_{2}-\mathrm{CH}_{2}\right)$, 4.48 (s, 4H, 2CH$\left.{ }_{2} \mathrm{Ph}\right), 7.14-7.32(\mathrm{~m}, 10 \mathrm{H}, \mathrm{Ar}-\mathrm{H}), 7.78-7.28$ (m, 4H, Ar-H), 7.93-7.90 (br s, 2H, NH; exchangeable with $\left.\mathrm{D}_{2} \mathrm{O}\right), 8.00-8.021(\mathrm{~m}, 2 \mathrm{H}, \mathrm{Ar}-\mathrm{H})$ and 8.26-8.29 (m, 2H, Ar-H); Anal. Calcd for $\mathrm{C}_{32} \mathrm{H}_{28} \mathrm{~N}_{6}$ (496.60): C, 77.39; H, 5.68; N, 16.92\%. Found: C, 76.29; H, 5.45; N, 16.65\%.

\subsection{General Method for the Synthesis of Hydrazino Pyrimidine Derivatives 14a,b}

A mixture of compound $9(0.25 \mathrm{~g}, 10 \mathrm{mmol})$ and 2-hydrazino-4-amino-5-cyano-6-arylpyrimidine $(10 \mathrm{mmol})$ in $n$-butanol $(30 \mathrm{~mL})$ was refluxed for 3 hours. The solvent was evaporated under vacuum. The solid obtained was filtered off and recrystallized from dioxane.

4-Amino-2-[N'-(4-benzylphthalazin-1-yl)hydrazino]-6-(4-methoxyphenyl)-pyrimidine-5-carbonitrile (14a). White crystals, 75\%, 0.16 g. mp 294-295 ${ }^{\circ} \mathrm{C}$; IR $\left(v_{\max }, \mathrm{cm}^{-1}\right): 3450,3210(\mathrm{NH}), 2206(\mathrm{CN}), 1634$ $(\mathrm{C}=\mathrm{N}) .{ }^{1} \mathrm{H}$ NMR $\left(\mathrm{DMSO}-d_{6}\right): \delta_{\mathrm{H}} 3.84\left(\mathrm{~s}, 3 \mathrm{H}, \mathrm{OCH}_{3}\right), 4.58\left(\mathrm{~s}, 2 \mathrm{H}, \mathrm{CH}_{2} \mathrm{Ph}\right), 7.10$ and $7.13(2 \mathrm{~d}$, $J=8.4 \mathrm{~Hz}), 2 \mathrm{H}, \mathrm{Ar}-\mathrm{H}), 7.17$ and $7.22(2 \mathrm{~d}, J=7.8 \mathrm{~Hz}, 2 \mathrm{H}, \mathrm{Ar}-\mathrm{H}), 7.37$ and $7.57(2 \mathrm{~d}, J=7.8 \mathrm{~Hz}, 4 \mathrm{H}$, Ar-H), 8.18 and $8.46(2 \mathrm{~d}, J=8.1 \mathrm{~Hz}, 2 \mathrm{H}, \mathrm{Ar}-\mathrm{H}), 8.684(\mathrm{~s}, 1 \mathrm{H}, \mathrm{Ar}-\mathrm{H}), 6.99$ and $7.88(\mathrm{~m}, 2 \mathrm{H}, \mathrm{Ar}-\mathrm{H})$, 6.250 (br s, 2H, $\mathrm{NH}_{2}$ ), 8.90 (br s, 2H, 2-NH); Anal. Calcd for $\mathrm{C}_{27} \mathrm{H}_{22} \mathrm{~N}_{8}$ (474.02): C, 68.34; H, 4.67; N, 23.612\%. Found: C, 68.34; H, 4.55; N, 23.54\%.

4-Amino-2-[N'-(4-benzylphthalazin-1-yl)hydrazino]-6-(4-chorophenyl)pyramidine-5-carbonitrile (14b). White crystals, $80 \%, 0.19$ g. mp 274-275 ${ }^{\circ} \mathrm{C}$; IR $\left(v_{\max }, \mathrm{cm}^{-1}\right)$ : 3380, $3270(\mathrm{NH}), 2192(\mathrm{CN}), 1638$ $(\mathrm{C}=\mathrm{N}) . \mathrm{MS}, m / z(\%)=478\left(\mathrm{M}^{+}\right.$, and $\left.\mathrm{M}+2\right), 462$ (19.22), 368 (14.61), 236 (12.68), 275 (75.31), 138 (36.35), 128 (46.12), 111 (29.49), 91(100); Anal. Calcd for $\mathrm{C}_{26} \mathrm{H}_{19} \mathrm{ClN}_{8}$ (478.01): C, 65.20; H, 4.00; N, 23.40\%. Found: C, 65.08; H, 3.87; N, 23.29\%. 
5-Benzyl-6,6a,12-triazobenzo[a]anthracen-7-one (15). A mixture of compound 9 (0.25 g, $10 \mathrm{mmol})$ and methyl anthranilate or anthranilic acid $(10 \mathrm{mmol})$ in absolute ethanol $(30 \mathrm{~mL})$ and 2 drops of triethylamine (TEA) was refluxed for 3 hours. The solvent was evaporated under vacuum. The solid obtained was filtered off and recrystallized from dioxane. Yellow crystals, 80\%, 0.16 g. mp 176-178 ${ }^{\circ} \mathrm{C}$; IR $\left(v_{\max }, \mathrm{cm}^{-1}\right): 1704(\mathrm{CO}) ; \mathrm{MS}, \mathrm{m} / z(\%)=337\left(\mathrm{M}^{+}, 66.92\right), 336$ (100) and 91 (13.09); Anal. Calcd for $\mathrm{C}_{22} \mathrm{H}_{15} \mathrm{~N}_{3} \mathrm{O}$ (337.12): C, 78.32; H, 4.48; N, 12.46\%. Found: C, 78.19; H, 4.37; N, 12.30\%.

[4-(4-Benzylphthalazin-1-ylamino)benzoylamino]acetic acid methyl ester (16). A mixture of compound $9(0.25 \mathrm{~g}, 10 \mathrm{mmol})$ and $p$-aminohippuric acid $(10 \mathrm{mmol})$ in methanol $(30 \mathrm{~mL})$ and three drops of TEA was refluxed for 3 hours. The solvent was evaporated under vacuum. The solid obtained was filtered off and recrystallized from dioxane. Yellow crystals, 90\%, 0.18 g. mp 165-167 ${ }^{\circ} \mathrm{C}$; IR $\left(v_{\max }, \mathrm{cm}^{-1}\right): 3110,3328(\mathrm{NH}), 1752(\mathrm{CO}), 1642(\mathrm{CONH}) .{ }^{1} \mathrm{H}$ NMR $\left(\mathrm{DMSO}-d_{6}\right): \delta_{\mathrm{H}} 3.67(\mathrm{~s}, 3 \mathrm{H}$, $\left.\mathrm{OCH}_{3}\right), 3.95(\mathrm{~d}, J=5.4 \mathrm{~Hz}), 1 \mathrm{H}$, non equivalent $\mathrm{CH}_{2}$ protons $), 4.04(\mathrm{~d}, J=5.4 \mathrm{~Hz}), 1 \mathrm{H}$, non equivalent $\mathrm{CH}_{2}$ protons), $4.73\left(\mathrm{~s}, 2 \mathrm{H}, \mathrm{PhCH}_{2}\right), 7.88$ and $8.006(2 \mathrm{~d}, J=8.7 \mathrm{~Hz}, 2 \mathrm{H}, \mathrm{Ar}-\mathrm{H}), 8.44(\mathrm{~d}$, $J=7.8 \mathrm{~Hz}, 1 \mathrm{H}, \mathrm{Ar}-\mathrm{H})$, three sets of multipletes at (7.24-7.30), (8.14-8.28), (8.93-9.01) (6H, Ar-H and $\mathrm{NH}$ ), 7.33 and 7.41 (AB-q, $J=7.2 \mathrm{~Hz}, 4 \mathrm{H}, \mathrm{Ar}-\mathrm{H}$ ), 10.80 (hump, $1 \mathrm{H}, \mathrm{NH}$, exchangeable by $\mathrm{D}_{2} \mathrm{O}$ ).; MS, $m / z(\%)=426\left(\mathrm{M}^{+}, 48.2\right), 425$ (100), 338 (27.8), 310 (5.4), 219 (2.7), 128 (5.4) and 91 (37.2).; Anal. Calcd for $\mathrm{C}_{15} \mathrm{H}_{22} \mathrm{~N}_{4} \mathrm{O}_{3}$ (426.46): C, 70.41; H, 5.20; N, 13.14\%. Found: C, 70.25; H, 5.07; N, 12.87\%.

Methyl 2-(4-(4-benzylphthalazin-1-ylamino)benzamido)-3-ethoxyacrolate (17). A mixture of compound $16(0.42 \mathrm{~g}, 1.0 \mathrm{mmol})$ and triethyl orthoformate $(0.14 \mathrm{~g}, 1.0 \mathrm{mmol})$ in acetic anhydride $(10 \mathrm{~mL})$ was refluxed for 5 hours, and then allowed to cool at room temperature and diluted with water (20 $\mathrm{mL})$. The solid obtained was filtered off and recrystallized from dioxane Yellow crystals, $60 \%, 0.17 \mathrm{~g}$. mp 175-177 ${ }^{\circ} \mathrm{C}$; IR ( $v_{\max }, \mathrm{cm}^{-1}$ ): 3060 (C-H olefinic ) 3366 (NH), 2942 (C-H aliphatic), 1752, 1666 (CO). ${ }^{1} \mathrm{H}$ NMR (DMSO- $\left.d_{6}\right): \delta_{\mathrm{H}} 2.08\left(\mathrm{t}, 3 \mathrm{H}, \mathrm{CH}_{2} \mathrm{CH}_{3}\right), 3.64\left(\mathrm{~s}, 3 \mathrm{H}, \mathrm{COOCH}_{3}\right), 4.01\left(\mathrm{q}, 2 \mathrm{H}, \mathrm{CH}_{2} \mathrm{CH}_{3}\right), 4.75$ (s, $2 \mathrm{H}, \mathrm{CH}_{2} \mathrm{Ph}$ ), (7.17-8.38) (sets of multiplets, $15 \mathrm{H}, \mathrm{Ar}-\mathrm{H}$ and $\left.2 \mathrm{NH}\right), 8.97(\mathrm{~s}, 1 \mathrm{H},=\mathrm{CH})$; Anal. Calcd for $\mathrm{C}_{28} \mathrm{H}_{26} \mathrm{~N}_{4} \mathrm{O}_{4}$ (482.52): C, 69.70; H, 5.43 N, 11.49\%. Found: C, 69.63; H, 5.23; N, 11.49\%.

2-(4-(4-Benzylphthalazin-1-ylamino)benzamido)acetamide (18). To compound 16 (0.42 g, 1.0 mmol) and distilled water and/or ammonium hydroxide, two drops of triethylamine (TEA) were added, and the mixture was refluxed in dioxane $(15 \mathrm{~mL})$ for 2 hours. The solvent was evaporated under vacuum. The solid obtained was filtered off and recrystallized from dioxane. Yellow crystals, 75\%, 0.19 g. mp 240$242{ }^{\circ} \mathrm{C}$; IR ( $\left.v_{\max }, \mathrm{cm}^{-1}\right): 3272,3354(\mathrm{NH}), 1734,1640(\mathrm{CO})$; MS, $m / z(\%)=411$ (55.9), 394 (20.7), 355 (59), 354 (100), 338 (48.0), 246 (32.8); Anal. Calcd for $\mathrm{C}_{24} \mathrm{H}_{21} \mathrm{~N}_{5} \mathrm{O}_{2}$ (411): C, 70.06; H, 5.14; N, $17.02 \%$. Found: C, 69.89; H, 5.07; N, 16.93\%.

\subsection{Antibacterial Activity}

The newly synthesized compounds were screened for their antimicrobial activities in vitro against two species of Gram-negative bacteria Pseudomonas aeruginosa (MTCC 741); Escherichia coli (NCTC-10410); and four Gram-positive bacteria, Bacillus cereus (ATGG 14579); Bacillus subtilis (MTCC 441); Bacillus sphaericus (MTCC 11); Staphylococcus (MTCC 96); and two fungus, Aspergillus ochraceus Wilhelm (AUCC-230); Penicillium chrysogenum Thom (AUCC-530). The 
activities of these compounds were tested using the disc diffusion method [20]. For bacteria and the paper disk diffusion method [21] for fungi. The area of zone of inhibition was measured using Ampicillin; tetracycline and norfloxacin $\left(30 \mu \mathrm{g} \mathrm{mL}{ }^{-1}\right)$ as standard antibiotic and mycostatin (30 $\mu \mathrm{g} \mathrm{mL}^{-1}$ ) was used as a reference antifungal. The tested compounds were dissolved in $N, N$-dimethylformamide (DMF) to give a solution of $1 \mathrm{mg} \mathrm{mL}^{-1}$. The inhibition zones were measured in millimeters at the end of an incubation period of 48 hours at $28^{\circ} \mathrm{C} . \mathrm{N}, \mathrm{N}$-dimethylformamide (DMF) showed no inhibition zone. Test results are shown in Table 1

Table 1. Antimicrobial activity of the new compounds.

\begin{tabular}{|c|c|c|c|c|c|c|c|c|}
\hline \multirow{2}{*}{$\begin{array}{c}\text { Compd. } \\
\text { No. }\end{array}$} & \multicolumn{7}{|c}{ Inhibition zone diameter in mm } \\
\cline { 2 - 9 } & $\begin{array}{c}\text { Gram-negative } \\
\text { bacteria }\end{array}$ & \multicolumn{3}{|c|}{ Gram-positive bacteria } & \multicolumn{2}{c|}{ Fungi } \\
\cline { 2 - 9 } & PA & EC & BC & BS & BS & SC & AOW & PCT \\
\hline $\mathbf{1}$ & 10 & 12 & 10 & 14 & 12 & 10 & 11 & 13 \\
\hline $\mathbf{2}$ & 14 & 10 & 12 & 11 & 10 & 13 & 10 & 11 \\
\hline $\mathbf{3}$ & 10 & 10 & 14 & 12 & 11 & 10 & 14 & 10 \\
\hline $\mathbf{4}$ & 13 & 10 & 12 & 13 & 10 & 12 & 10 & 12 \\
\hline $\mathbf{5}$ & 11 & 14 & 10 & 13 & 14 & 13 & 12 & 13 \\
\hline $\mathbf{6}$ & 14 & 10 & 13 & 10 & 12 & 14 & 11 & 14 \\
\hline $\mathbf{7}$ & 10 & 12 & 15 & 12 & 10 & 11 & 10 & 11 \\
\hline $\mathbf{8}$ & 12 & 13 & 10 & 14 & 10 & 14 & 10 & 10 \\
\hline $\mathbf{1 0 a}$ & 16 & 15 & 14 & 15 & 15 & 16 & 17 & 15 \\
\hline $\mathbf{1 0 b}$ & 18 & 19 & 18 & 17 & 15 & 19 & 14 & 17 \\
\hline $\mathbf{1 0 c}$ & 16 & 16 & 19 & 15 & 18 & 14 & 17 & 18 \\
\hline $\mathbf{1 0 d}$ & 15 & 17 & 16 & 19 & 17 & 15 & 16 & 14 \\
\hline $\mathbf{1 0 e}$ & 17 & 16 & 14 & 17 & 19 & 16 & 19 & 19 \\
\hline $\mathbf{1 0 f}$ & 16 & 15 & 17 & 18 & 17 & 14 & 15 & 17 \\
\hline $\mathbf{1 0 g}$ & 18 & 15 & 15 & 16 & 16 & 17 & 18 & 16 \\
\hline $\mathbf{1 0 h}$ & 19 & 18 & 14 & 15 & 17 & 15 & 14 & 19 \\
\hline $\mathbf{1 0}$ & 17 & 16 & 17 & 19 & 18 & 15 & 17 & 15 \\
\hline $\mathbf{1 0 j}$ & 18 & 15 & 19 & 16 & 18 & 15 & 16 & 14 \\
\hline $\mathbf{1 1}$ & 12 & 10 & 14 & 12 & 10 & 14 & 10 & 11 \\
\hline $\mathbf{1 2 a}$ & 20 & 22 & 20 & 19 & 20 & 22 & 20 & 20 \\
\hline $\mathbf{1 2 b}$ & 20 & 23 & 22 & 24 & 24 & 20 & 20 & 20 \\
\hline $\mathbf{1 3}$ & 22 & 20 & 23 & 22 & 23 & 20 & 24 & 22 \\
\hline $\mathbf{1 4 a}$ & 23 & 22 & 24 & 23 & 22 & 24 & 23 & 23 \\
\hline $\mathbf{1 4 b}$ & 24 & 20 & 20 & 20 & 22 & 24 & 20 & 22 \\
\hline $\mathbf{1 5}$ & 19 & 22 & 23 & 22 & 24 & 20 & 20 & 21 \\
\hline $\mathbf{1 6}$ & 18 & 17 & 18 & 17 & 19 & 18 & 16 & 17 \\
\hline $\mathbf{1 7}$ & 17 & 19 & 16 & 19 & 17 & 19 & 15 & 16 \\
\hline $\mathbf{1 8}$ & 15 & 18 & 15 & 17 & 18 & 16 & 18 & 15 \\
\hline Ampicillin & 22 & 22 & 22 & 22 & 22 & 22 & - & - \\
\hline Tetracycline & 20 & 20 & 20 & 20 & 20 & 20 & - & - \\
\hline Norfloxacin & 25 & 25 & 25 & 25 & 25 & 25 & - & - \\
\hline Mycostatin & - & - & - & - & - & - & 20 & 20 \\
\hline
\end{tabular}

${ }^{a} c=1 \mathrm{mg} \mathrm{mL}{ }^{-1}$ of new compounds in DMF. Microorganisms: Pseudomonas aeruginosa (MTCC 741); Escherichia coli (NCTC-10410); Bacillus cereus (ATGG 14579); Bacillus subtilis (MTCC 441); Bacillus sphaericus (MTCC 11); Staphylococcus (MTCC 96); Aspergillus ochraceus Wilhelm (AUCC-230); Penicillium chrysogenum Thom (AUCC-530). 


\subsection{Antibacterial Activities}

The screening results indicate that compounds $\mathbf{2 - 8}$ and $\mathbf{1 1}$ show weaker inhibitory activity than the standard drugs, while compounds 15-18 are moderately inhibitory, compared to the standard drugs. Compounds 10a-j, 12, 13 and 14a,b showed nearly the same inhibition activity asn antibacterial activity (Table 1). It clear that decomposition of chloride atom at C-1 of 4-benzyl-1-chlorophthalazine with $\mathrm{N}$-nucleophiles is responsible for the antimicrobial activities.

\section{Conclusions}

The results from this screening demonstrated that replacement of the hydrogen atom attached to the phthalazine nucleus at $\mathrm{N}-1$ with amino derivatives (compounds 10a-j), diimino derivatives (compounds 12a,b-13) and pyrimidine derivatives $(\mathbf{1 4 a}, \mathbf{b})$ resulted in spectrum if moderate antibacterial activity against all tested Gram positive and Gram negative fungi.

\section{References}

1. Ito, S.; Yamaguchi, K.; Komoda, Y. Structural confirmation of the nitration product of the 1(2H)-phthalazinone as the 2-Nitro-1(2H)-phthalazinone. Chem. Pharm. Bull. 1992, 40, 3327-3329.

2. Haack, T.; Fattori, R.; Napoletano, M.; Pellacini, F.; Fronza, G.; Raffaini, G.; Ganazzoli, F. Phthalazine PDE IV inhibitors: Conformational study of some 6-methoxy-1,4-disubstituted derivatives. Bioorg. Med. Chem. 2005, 13, 4425-4433.

3. Heinisch, G.; Frank, H. In Progress in Medicinal Chemistry; Ellis, G.P., Luscombe, D.K., Eds; Elsevier: Amesterdam, The Netherlands, 1990; Volume 27, pp. 1-49.

4. Heinisch, G.; Frank, H. In Progress in Medicinal Chemistry; Ellis, G.P., Luscombe, D.K., Eds; Elsevier: Amesterdam, The Netherlands, 1992; Volume 29, pp. 141-1483.

5. Melikian, A.; Schiewer, G.; Chambon, J.P.; Wermuth, C.G. Condensation of muscimol or thiomuscimol with aminopyridazines yields GABA-A antagonists. J. Med. Chem. 1992, 35, 4092-4197.

6. Napoletano, M.; Norcini, G.; Pellacini, F.; Morazzoni, G.; Ferlenga, P.; Pradella, L. Phthalazine PDE4 inhibitiors. Part 2: The synthesis and biological evolution of 6-methoxy-1,4-disubstituted derivatives. Bioorg. Med. Chem. Lett. 2001, 11, 33-37.

7. Kikuchi, K.; Nagano, T.; Hayakawa, H.; Hirata, Y.; Hirobe, M. Detection of nitric oxide production from a perfused organ by a luminol-hydrogen peroxide system. Anal. Chem. 1999, 22, 5131-5136.

8. Arakawa, H.; Ishida, J.; Yamaguchi, M.; Nakamura, M. Chemiluminescent products of reaction between $\alpha$-Keto acids and 4,5-diaminophthalhydrazide. Chem. Pharm. Bull. 1990, 38, 3491-3499.

9. Arakawa, H.; Ishida, J.; Yamaguchi, M.; Nakamura, M. New chemiluminogenic substrate for N-Acetyl- $\beta$-D-glucosaminidase, 4'-(6'-diethylamino-benzofuranyl)phthalylhydrazido- $N$-acetyl- $\beta$ D-glucosaminide. Chem. Pharm. Bull. 1991, 39, 411-419.

10. Samoto, K.; Ohkura, Y. Ring expansion reaction of 1,3-dithiolanes and 1,3-oxathiolanes using tellurium tetrachloride. Chem. Lett. 1990, 19, 1323. 
11. Tsoungas, P.G.; Searcey, M. A convenient access to benzo-substituted phthalazines as potential precursors to DNA intercalators. Tetrahedron Lett. 2001, 42, 6589-6592.

12. Sivakumar, R.; Gnanasam, S.K.; Ramachandran, S.; Leonard, J.T. Pharmacological evaluation of some new 1-substituted-4-hydroxy-phthalazines. Eur. J. Med. Chem. 2002, 37, 793-801.

13. Coelho, A.; Sotelo, E.; Fraiz, N.M.; Yanez, M.; Laguna, R.; Canob, E.; Ravina, E. Pyridazines. Part 36: Synthesis and antiplatelet activity of 5-substituted-6-phenyl-3(2H)-pyridazinones. Bioorg. Med. Chem. Lett. 2004, 14, 321-324.

14. Demirayak, S.; Karaburun, A.C.; Beis, R. Some pyrrole substituted aryl pyridazinone and phthalazinone derivatives and their antihypertensive activities. Eur. J. Med. Chem. 2004, 39, 1089-1095.

15. Dogruer, D.S.; Sahin, M.F.; Unlu, S.; Ito, S. Studies on some 3(2H)-pyridazinone derivatives with antinociceptive activity. Arch. Pharm. 2000, 333, 79-86.

16. Dogruer, D.S.; Sahin, M.F.; Kupeli, E.; Yesilada, E. Synthesis and analgesic and anti-inflammatory activity of new pyridazinones. Turk. J. Chem. 2003, 27, 727-738.

17. Dogruer, D.S.; Sahin, M.F.; Kupeli, E.; Yesilada, E. Synthesis of new 2-[1(2H)-phthalazinon-2yl]acetamide and 3-[1(2H)-phthalazinon-2-yl]propanamide derivatives as antinociceptive and anti-inflammatory agents. Arch. Pharm. 2004, 337, 303-310.

18. Sonmez, M.M.; Berber, I.; Akbas, E. Synthesis, antibacterial and antifungal activity of some new pyridazinone metal complexes. Eur. J. Med. Chem. 2006, 41, 101-105.

19. Condensed Pyridazines Including Cinnolines and Phthalazines; Castle, N.R., Ed.; Wiley-Intersicence: New York, NY, USA, 1973; p. 383.

20. European Committee for Antimicrobial Susceptibility Testing (EUCAST) of the European Society of Clinical Microbiology and Infectious Diseases (ESCMID). Determination of minimum inhibitory concentrations (MICs) of antibacterial agents by agar dilution. Clin. Microbiol. Infect. 2000, 6, 509-515.

21. National Committee for Clinical Laboratory Standards. Methods for dilution antimicrobial susceptibility tests for bacteria that grow aerobically, 5th ed.; Approved Standard M7-A5. NCCLS: Wayne, PA, USA, 2000.

(C) 2011 by the authors; licensee MDPI, Basel, Switzerland. This article is an open access article distributed under the terms and conditions of the Creative Commons Attribution license (http://creativecommons.org/licenses/by/3.0/). 